\title{
Unexpected Participation of Intercalated Cells in Renal Inflammation and Acute Kidney Injury
}

\author{
Sylvie Breton ${ }^{a} \quad$ Maria Agustina Battistone ${ }^{b, c}$ \\ aResearch Center, Centre Hospitalier Universitaire de Québec, Université Laval, Québec, QC, Canada; brogram \\ in Membrane Biology/Nephrology Division, Massachusetts General Hospital, Boston, MA, USA; ${ }^{C}$ Harvard Medical \\ School, Boston, MA, USA
}

\section{Keywords}

Immune cell infiltration · Neutrophils · Monocytes · Tubular injury · Sterile inflammation · Innate immunity

\begin{abstract}
Epithelial cells constitute the 1st line of defense against pathogens, and their participation in innate immunity is rapidly emerging. In this mini-review, we discuss the noncanonical role of renal intercalated cells (ICs) in pathogen defense and in the initiation of sterile inflammation. This last function has strong implications in the onset of acute kidney injury $(A K I)$, a potentially fatal medical complication that is seen in hospitalized patients. AKI is associated with inflammation, and it is often diagnosed only after the kidneys have suffered significant and often irreversible damage. While examining the regulation of proton secretion by type A ICs (A-ICs), we unexpectedly found high expression of the pro-inflammatory purinergic receptor $\mathrm{P} 2 \mathrm{Y} 14$ in these cells. This receptor is located on the apical surface of A-ICs and binds UDP-glucose (UDP-GIc), a danger-associated molecular pattern molecule released from injured cells that is filtered by the glomeruli and is concentrated in the collecting duct lumen. UDP-GIc activates P2Y14 in A-ICs and triggers the production of chemokines that attract pro-inflammatory immune cells into the kidney stroma and aggravate ischemia-induced proximal tu-
\end{abstract}

bule injury. Inhibition of P2Y14 or deletion of its gene specifically in ICs in a murine model of ischemia-reperfusion injury attenuated these effects. Thus, together with their previously recognized role in pathogen defense, A-ICs are now recognized as sensors and mediators of renal sterile inflammation that participate in the onset of AKI. Blocking the UDPGlc/P2Y14 pathway in A-ICs provides new insights into the development of novel AKI therapeutics.

두 2021 S. Karger AG, Base

\section{Introduction}

The kidneys maintain systemic water, salt, and $\mathrm{pH}$ balance while eliminating toxins. Several epithelial cell types play specific roles in each of these functions. Type A intercalated cells (A-ICs) are located along the collecting duct $(C D)$, where they regulate and maintain acid-base balance by adjusting proton secretion through a critical

Contribution from the AKI and CRRT 2021 Symposium at the 26th International Conference on Advances in Critical Care Nephrology, A Virtual/Hybrid Event from San Diego, CA, USA, February 28-March 5, 2021. This symposium was supported in part by the NIDDK funded University of Alabama at Birmingham-University of California San Diego O'Brien Center for Acute Kidney Injury Research (P30DK079337). karger@karger.com

www.karger.com/nef

Karger ${ }^{\prime \prime}=$
(C) 2021 S. Karger AG, Basel

(
Correspondence to:

Sylvie Breton, sylvie.breton@ crchudequebec.ulaval.ca 
enzyme, the proton pumping V-ATPase, located in their apical membrane [1]. However, it is now recognized that, in addition to this important physiological role, A-ICs are critical sensors and mediators of renal inflammation [26]. While A-ICs participate in the renal defense against urinary infections by triggering pathogen-induced inflammation, they can also initiate harmful inflammation in the absence of infection. This sterile inflammation occurs following activation of the pro-inflammatory P2Y14 receptor located on their apical surface. In this mini-review, we will describe the noncanonical pro-inflammatory role of A-ICs and their participation in the onset of acute kidney injury (AKI).

\section{AKI and Renal Inflammation}

Regardless of the cause of hospitalization, patients admitted to the intensive care unit are susceptible to developing AKI. AKI can lead to chronic kidney disease and end-stage renal disease, and it induces longer hospitalization [7]. AKI patients are also at risk of multiple organ failure and death. The onset of AKI causes no symptoms, and AKI is often detected only after significant kidney injury has occurred. On a yearly basis, $>4$ million people in North America suffer from AKI, and 300,000 people die from it. Diagnostic methods for AKI are inadequate, and there is no specific treatment for this potentially fatal condition [8]. In this context, the field is in dire need of new diagnostics and treatments administered, while renal injury can be prevented.

AKI is frequent after cardiac surgery requiring cardiopulmonary bypass, myocardial infarction, and sepsis [7]. AKI following these medical conditions is the consequence of a direct insult to the kidney or a distant organ injury, and it is often associated with renal ischemia. Ischemic AKI is accompanied by infiltration of circulating pro-inflammatory immune cells into the kidney stroma [7]. This sets up an inflammatory cascade that enhances kidney injury.

\section{Renal Pathogen-Induced and Sterile Inflammation}

Renal inflammation occurs in both septic and nonseptic AKI. The innate immune response is triggered by alert molecules known as pathogen-associated molecular patterns that are released by bacteria or danger-associated molecular patterns (DAMPs) that are released by the host cells in response to injury $[7,9]$. Activation of pattern recognition receptors by DAMPs or pathogen-associated molecular patterns induces the production of chemokines, which attract neutrophils and monocytes into the tissue [7]. These newly recruited cells produce cytotoxic

Role of Intercalated Cells in Renal Inflammation substances such as reactive oxygen species. They also induce microvasculature congestion, which ultimately impairs renal blood flow, creating sustained ischemia, and further contributes to increasing kidney damage [7]. In mice, neutrophils and monocytes are recruited rapidly (within $2 \mathrm{~h}$ ) into the kidney stroma after renal ischemiareperfusion injury (IRI) [3]. Blockade of this very 1st step in the inflammatory cascade, thus, offers strong therapeutic potential.

\section{Role of A-ICs in the Renal Defense Against Pathogens}

Previous studies have shown the participation of AICs in the innate immunity against pathogens [4-6]. AICs recognize and bind urinary pathogens, in particular uropathogenic Escherichia coli, via activation of both TLR4-dependent and TLR4-independent pathways [4]. A-ICs then acidify the urine and secrete the bacteriostatic protein lipocalin 2 (also known as NGAL) [5]. Mice deficient in A-ICs following deletion of the transcription factor $T c f c p 2 l 1$ had reduced bacterial clearance, further supporting the critical role of these cells in pathogen defense. In a more recent study, ICs were shown to have characteristics of phagocytic cells such as myeloid-derived macrophages, since they phagocytized uropathogenic Escherichia coli in vivo and then acidified their phagolysosomes possibly via V-ATPase activity [6]. While these studies collectively identified ICs as key players in the defense against pathogens, we showed that A-ICs can also participate in the onset of renal inflammation in the absence of infection, via cell-cell cross talk involving the DAMP molecule, uridine diphosphate glucose (UDPGlc), a P2Y14 receptor ligand.

\section{Role of ICs in Kidney Sterile Inflammation}

Several DAMPs were shown to be associated with AKI. These include high-mobility group protein B1, ATP, DNA, mitochondrial DNA, and UDP-Glc [3, 9]. Intracellularly, UDP-Glc participates in glycosylation reactions, but in disease state it becomes a potent agonist of P2Y14 when secreted from injured cells [10]. Unlike other purinergic receptors, $\mathrm{P} 2 \mathrm{Y} 14$ is activated by UDP-Glc and not by other nucleotides such as ATP, ADP, or UTP. UDPGlc is released as cargo via the constitutive secretory pathway and via $\mathrm{Ca}^{2+}$-regulated exocytosis of Golgi-derived vesicles. Nonvesicular mechanisms that contribute to the release of ATP may also participate in the secretion of UDP-Glc [10]. While UDP-Glc release is activated in injured cells, it is unlikely that the novo UDP-Glc production would increase, since metabolism pathways are often stopped under stressed conditions. Whereas most nucle- 
Fig. 1. Recruitment of neutrophils next to PTs and ICs after renal IRI and attenuation of this recruitment by PPTN. a Kidney sections were labeled using the neutrophil marker Ly6G (red) and the PT marker AQP1 (green). While no neutrophils were detected in the Sham group, many neutrophils were detected close to medullary PTs $24 \mathrm{~h}$ post-IRI (IRI $24 \mathrm{~h}$; arrows). In the PPTN-treated group, very few neutrophils were detected $24 \mathrm{~h}$ post-IRI (IRI $24 \mathrm{~h}$ PPTN; arrow). b Kidney sections were labeled using Ly6G (red: arrows) and the ICspecific marker V-ATPase B1 subunit (B1 V-ATPase, green; arrowheads). No neutrophils were seen in the Sham group. At $24 \mathrm{~h}$ post-IRI, many Ly6G-positive neutrophils (IRI 24 h) were detected, while in the PPTN group, very few neutrophils were detected $24 \mathrm{~h}$ post-IRI (IRI $24 \mathrm{~h}$ PPTN). Scale bars = $10 \mu \mathrm{m}$. Figure modified from [3]. ICs, intercalated cells; IRI, ischemia-reperfusion injury; PTs, proximal tubules; PPTN, 4,7-disubstituted 2-naphthoic acid derivative.

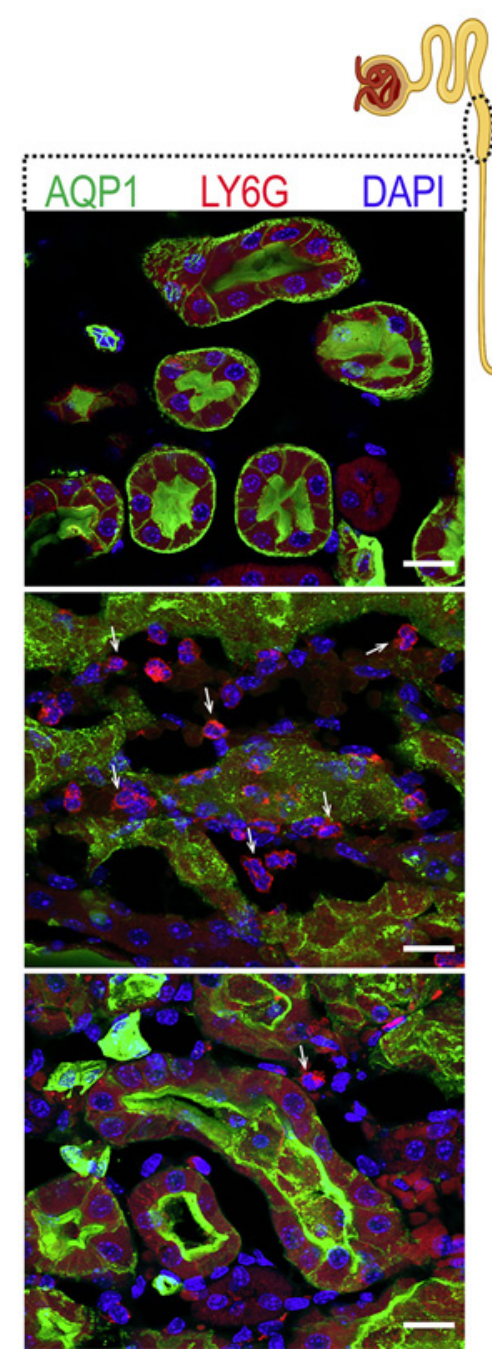

a

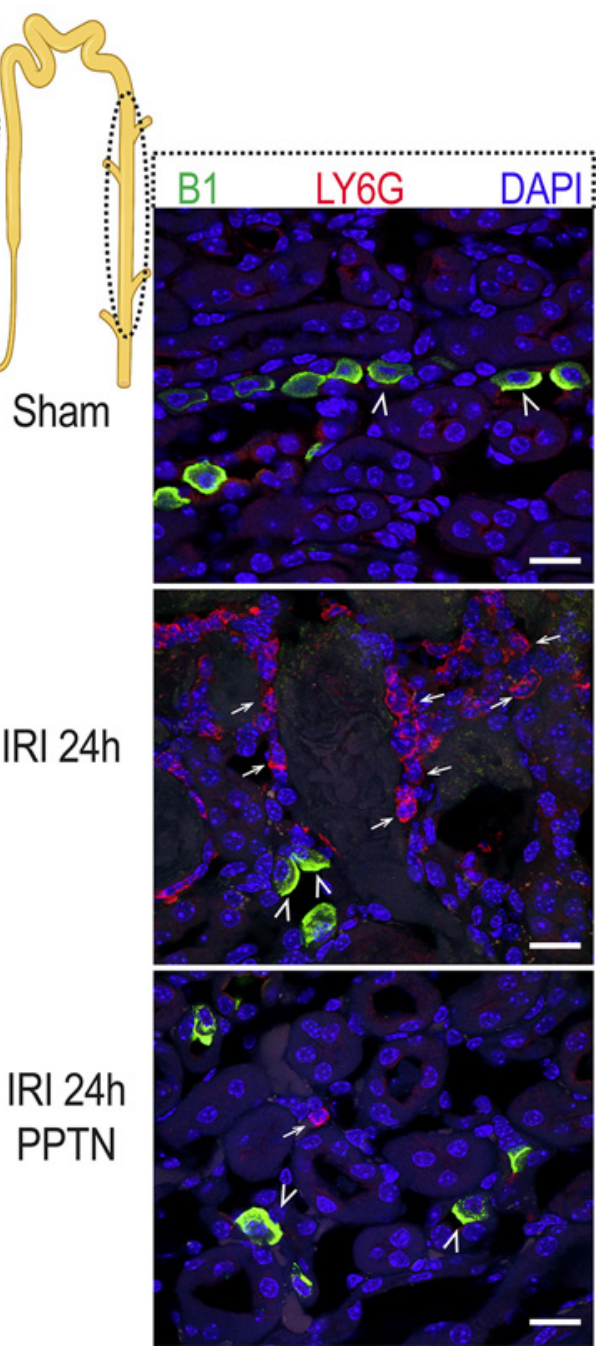

b otides, such as ATP, are rapidly degraded by ectonucleotidases after their release, extracellular UDP-Glc is highly stable. Thus, the release of UDP-Glc from stressed cells could lead to elevated extracellular concentrations, leading to P2Y14 activation. P2Y14 is expressed in epithelial cells in the lung, uterus, and kidney $[2,3,11,12]$.

Elevated UDP-Glc levels and neutrophil infiltration were observed in sputum samples from cystic fibrosis patients compared to that in control subjects. In addition, UDP-Glc intratracheal instillation and injection into the uterus in mice promoted neutrophil migration into the lung and endometrium, respectively, indicating a link between UDP-Glc and inflammation [11, 12]. We recently showed that A-ICs are sensors of UDP-Glc and mediate neutrophil and monocyte renal recruitment via activation of P2Y14 $[2,3]$. Another proposed mechanism for the participation of ICs in sterile inflammation is through their secretion of NGAL, which occurs following renal ischemia [5].

\section{The UDP-Glucose/P2Y14 Pathway: A Potential}

Diagnostic-Therapeutic Combination for AKI

Transcriptomic analysis of EGFP expressing A-ICs isolated from transgenic mice and single-cell RNA sequencing unexpectedly identified P2Y14 as one of the most expressed genes in these cells $[3,13]$. No P2Y14 expression was detected in other renal tubule epithelial cells $[2,13]$. We showed that P2Y14 is located on the apical 
surface of A-ICs, where it is in contact with the CD luminal fluid, and that a single i.v. injection of UDP-Glc triggered pro-inflammatory chemokine expression in A-ICs [2]. In this 1st study, we detected recruitment of neutrophils to the renal medulla 2 days after the injection, identifying A-ICs as sensors, mediators, and effectors of renal sterile inflammation via activation of P2Y14 by UDP-Glc.

We then used the IRI model to explore the novel role of A-ICs in the pathogenesis of the renal inflammation that leads to kidney injury [3]. RNA sequencing revealed the upregulation of pro-inflammatory factors including the P2Y14 itself in isolated ICs as early as $2 \mathrm{~h}$ post-surgery [3]. In the same study, we found that blocking P2Y14 with a small molecule (4,7-disubstituted 2-naphthoic acid derivative $[\mathrm{PPTN}]$ ) or ablation of the gene encoding P2Y14 in ICs specifically (1) inhibited the increase in chemokine expression induced by IRI in ICs; (2) reduced neutrophil and monocyte infiltration into the kidney (analyzed by flow cytometry); (3) reduced the extent of kidney dysfunction following IRI (based on serum creatinine [SCr], blood urea nitrogen, and albuminuria); and (4) attenuated proximal tubule (PT) damage. Figure 1 shows that in nontreated mice subjected to IRI, many neutrophils were recruited into the outer medulla, where PTs and ICs are located (IRI $24 \mathrm{~h}$ ). Fewer neutrophils were seen in PPTNtreated mice (Fig. 1; IRI $24 \mathrm{~h}$ PPTN) and IC-specific P2Y14 KO mice [3]. Our study, therefore, indicated that P2Y14 participates in the 1st inflammatory steps associated with ischemic AKI and that blocking P2Y14 reduces the impact of IRI.

Finally, in a longitudinal pilot study, urine UDP-Glc levels correlated with the incidence of AKI in intensive care unit patients [3]. An even stronger correlation was observed in cardiac surgery patients. This study, therefore, identified UDP-Glc as a promising actionable biomarker and a predictor of AKI, and P2Y 14 as a potential therapeutic target, which might be used to prevent or alleviate ischemic AKI.

Overall, these results provided new insight on how not only a local insult to the kidney but also an injury to a remote organ communicates damage to the kidney via activation of P2Y14 located in A-ICs. In addition to damaged PTs during renal ischemia, cardiomyocytes during myocardial infarction or cardiac surgery, or cells damaged by sepsis might also release UDP-Glc. UDP-Glc would then become a circulating mediator that initiates intra- and interorgan cross talk. Among other organs, the kidneys are particularly susceptible to circulating factors because one of their main roles is to remove metabolites such as UDP-Glc from the blood. During this process, serum UDP-Glc is filtered by the glomeruli and delivered to the CD lumen, where it ultimately causes renal inflammation and AKI. This sets up UDP-Glc apart from most current biomarkers because it is not only a bystander of AKI but it also contributes to kidney injury. Detection of UDP-Glc in the urine of patients might then translate into a truly actionable intervention, such as the use of a specific P2Y14 blocker that would inhibit its action and ultimately alleviate or even prevent AKI.

The lack of an accurate diagnostic and effective treatment for AKI has negatively impacted the nephrology field. The marker currently used for the detection of AKI is elevated $\mathrm{SCr}[8,14]$. However, $\mathrm{SCr}$ is widely recognized as a poor and lagging marker because significant loss of kidney function is required to induce its detectable rise. In fact, subclinical AKI can occur without an increase in SCr, through the renal reserve where unaffected tubules provide a compensatory mechanism [8]. Nevertheless, even subclinical AKI is associated with poor outcomes [15]. SCr also depends on several factors that are not related to AKI, such as age, muscle mass, medication, and general medical conditions. In the setting of cardiac surgery, hemodilution during cardiopulmonary bypass tends to decrease SCr, further impairing AKI detection.

In addition, the absence of an appropriate AKI biomarker has slowed down the development of novel therapeutics for AKI. How could a clinical study be successful if the disease it is trying to cure is not accurately detectable? How could a novel biomarker be evaluated if the current SCr "gold standard" is imperfect? It is, therefore, possible that a novel AKI biomarker or therapy would remain unnoticed and be unfairly discarded because of a flaw in the "gold standard" to which it is compared [14]. In this context, the availability of a causal biomarker that is associated with a mean to block its action might provide a promising new avenue to a field that is in urgent need of a diagnostic/therapeutic solution.

\section{Summary and Conclusion}

Our current model is illustrated in Figure 2. Medullary PTs are the most affected segments following IRI, and we postulated that injured PT cells release UDP-Glc. UDPGlc is then concentrated in the CD lumen to a critical point where it initiates local inflammation, via activation of P2Y14 in A-ICs. This was supported by the elevation of urinary UDP-Glc that we observed $2 \mathrm{~h}$ post-IRI in mice [3]. Newly recruited neutrophils and monocytes "attack" PT cells, inducing further injury and preventing the re- 
Fig. 2. Proposed mechanism of action of UDP-Glc in mediating renal inflammation following IRI. UDP-Glc is released from injured PT cells and is then delivered to the lumen of the kidney $\mathrm{CD}$, where it reaches higher levels due to the concentrating ability of the kidney. UDP-Glc binds to the $\mathrm{P} 2 \mathrm{Y} 14$ receptor located on the apical surface of A-ICs. This receptor-ligand interaction stimulates the production of pro-inflammatory chemokines by ICs, which attract neutrophils and monocytes from the circulation into the kidney stroma. The newly recruited immune cells aggravate renal tubular injury and prevent the repair process. CD, collecting duct; IRI, ischemiareperfusion injury; UDP-Glc, uridine diphosphate glucose; PT, proximal tubule; AICs, type A intercalated cells; ICs, intercalated cells; AKI, acute kidney injury.

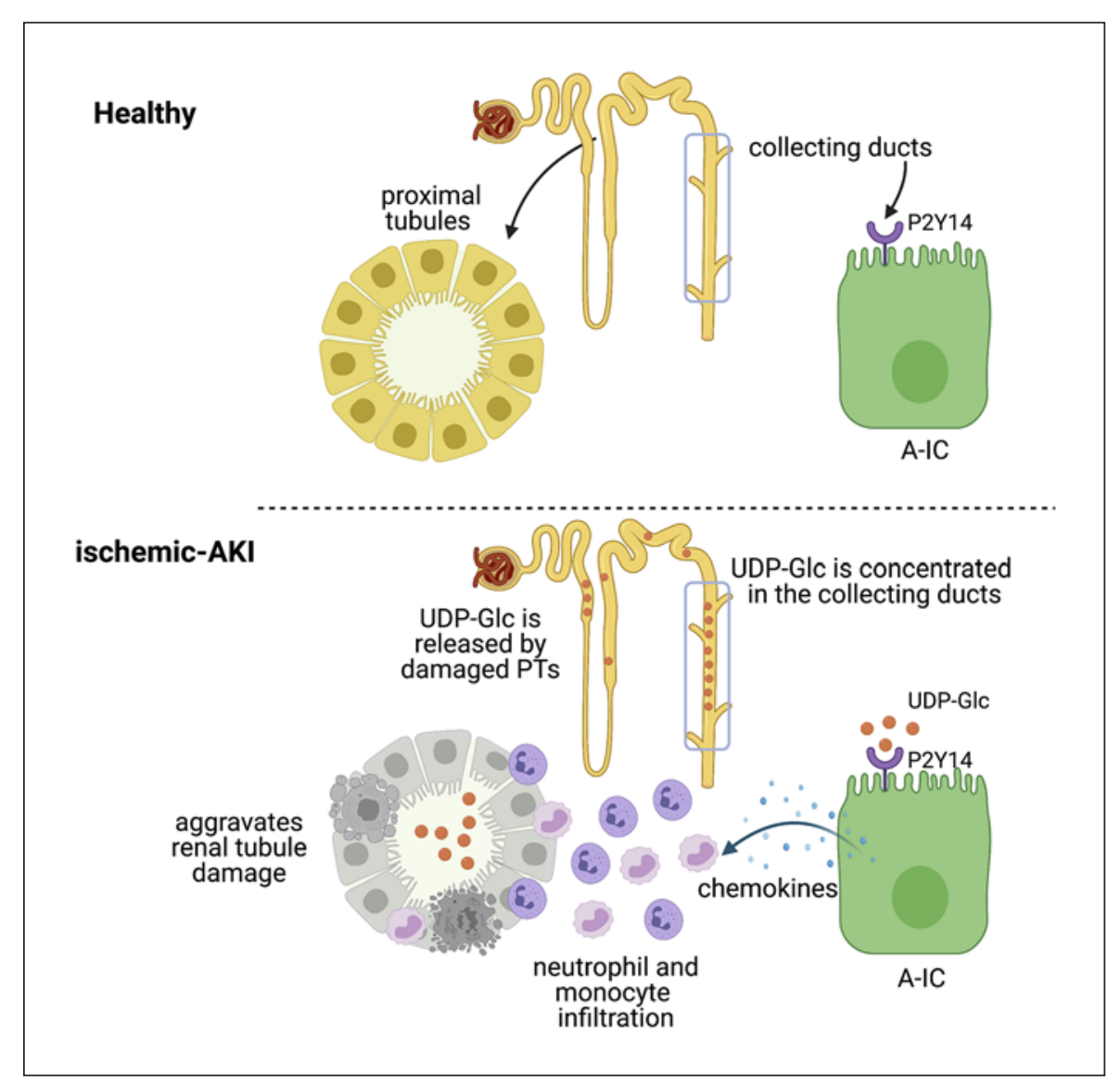

pair process. Of note, the cross talk between PTs and ICs is facilitated by the unique architecture of the kidney medulla, which harbors PTs, CDs, and blood vessels that run in parallel with each other. The discovery that professional proton-secreting ICs in the kidney can participate in renal inflammation and pathogen defense illustrates the importance of conducting fundamental research to promote biomedical progress. While examining the regulation of ICs in the context of their proton secretory activity, we found their contribution to sterile inflammation through the UDP-Glc/P2Y14 signaling pathway. AKI is a multifactorial medical complication, and a patient-centered approach would favor the development of novel therapeutics. In this context, the availability of biomarkers that are linked to the therapy being tested would allow enrichment strategies and intervention to a specific group of patients who are more likely to benefit from treatment. Because UDP-Glc is a causal marker of AKI that induces early renal inflammation, it would provide such an advantage by using a specific P2Y14 blocker. Future clinical investigations will be required to determine the potential therapeutic benefits of inhibiting the UDP-Glc/P2Y14 pathway to alleviate or even prevent hospital-acquired AKI.

\section{Conflict of Interest Statement}

Maria Agustina Battistone has no conflicts of interest to declare. Sylvie Breton is a cofounder of Kantum Pharma (previously "Kantum Diagnostics Inc."), a company developing a diagnostic and therapeutic combination to prevent and treat AKI. Sylvie Breton is an inventor on a patent (US Patent $10,088,489$ ) covering technology that has been licensed to the company through Massachusetts General Hospital (MGH). Sylvie Breton's interests were reviewed and are managed by MGH in accordance with their conflict-of-interest policies.

\section{Funding Sources}

These studies were supported by National Institutes of Health HD040793, DK097124, and 5U5HL119145 Grants (to S.B.), a Lalor Foundation Grant and National Institutes of Health RO1 HD104672-01 Grant (to M.A.B.), and a gift from the Danone 
Research Institute. S.B. is a recipient of the Canadian Research Chair in Epithelial Dynamics of the Kidney and Reproductive Organs.

\section{Author Contributions}

M.A.B. and S.B. designed and wrote the manuscript.

\section{References}

1 Eaton AF, Merkulova M, Brown D. The H+ATPase (V-ATPase): from proton pump to signaling complex in health and disease. Am J Physiol Cell Physiol. 2021;320:C392-414.

2 Azroyan A, Cortez-Retamozo V, Bouley R, Liberman R, Ruan YC, Kiselev E, et al. Renal intercalated cells sense and mediate inflammation via the P2Y14 receptor. PLoS One. 2015; 10:e0121419.

3 Battistone MA, Mendelsohn AC, Spallanzani RG, Allegretti AS, Liberman RN, Sesma J, et al. Proinflammatory P2Y14 receptor inhibition protects against ischemic acute kidney injury in mice. J Clin Invest. 2020;130:373449.

4 Chassin C, Goujon JM, Darche S, du Merle L, Bens M, Cluzeaud F, et al. Renal collecting duct epithelial cells react to pyelonephritisassociated Escherichia coli by activating distinct TLR4-dependent and -independent inflammatory pathways. J Immunol. 2006;177: 4773-84.
5 Paragas N, Kulkarni R, Werth M, SchmidtOtt KM, Forster C, Deng R, et al. a-Intercalated cells defend the urinary system from bacterial infection. J Clin Invest. 2014;124:2963-76.

6 Saxena V, Gao H, Arregui S, Zollman A, Kamocka MM, Xuei X, et al. Kidney intercalated cells are phagocytic and acidify internalized uropathogenic Escherichia coli. Nat Commun. 2021;12(1):2405.

7 Singbartl K, Formeck CL, Kellum JA. Kidneyimmune system crosstalk in AKI. Semin Nephrol. 2019;39:96-106.

8 Moledina DG, Parikh CR. Phenotyping of acute kidney injury: beyond serum creatinine. Semin Nephrol. 2018;38:3-11.

9 Schaefer L. Complexity of danger: the diverse nature of damage-associated molecular patterns. J Biol Chem. 2014;289:35237-45.

10 Lazarowski ER, Harden TK. UDP-sugars as extracellular signaling molecules: cellular and physiologic consequences of $\mathrm{P} 2 \mathrm{Y} 14$ receptor activation. Mol Pharmacol. 2015;88:151-60.
11 Arase T, Uchida H, Kajitani T, Ono M, Tamaki K, Oda $\mathrm{H}$, et al. The UDP-glucose receptor P2RY14 triggers innate mucosal immunity in the female reproductive tract by inducing IL-8. J Immunol. 2009;182:7074-84.

12 Sesma JI, Weitzer CD, Livraghi-Butrico A, Dang H, Donaldson S, Alexis NE, et al. UDPglucose promotes neutrophil recruitment in the lung. Purinergic Signal. 2016;12(4):62735.

13 Chen L, Lee JW, Chou CL, Nair AV, Battistone MA, Păunescu TG, et al. Transcriptomes of major renal collecting duct cell types in mouse identified by single-cell RNA-seq. Proc Natl Acad Sci U S A. 2017;114:E998998.

14 Waikar SS, Betensky RA, Emerson SC, Bonventre JV. Imperfect gold standards for biomarker evaluation. Clin Trials. 2013;10:696700.

15 Ronco C, Kellum JA, Haase M. Subclinical AKI is still AKI. Crit Care. 2012;16(3):313. 